\title{
Efficient sliding spotlight SAR raw signal simulation of extended scenes
}

Wei $\mathrm{Xu}^{1^{*}}$, Pingping Huang ${ }^{2}$ and Yunkai Deng ${ }^{1}$

\begin{abstract}
Sliding spotlight mode is a novel synthetic aperture radar (SAR) imaging scheme with an achieved azimuth resolution better than stripmap mode and ground coverage larger than spotlight configuration. However, its raw signal simulation of extended scenes may not be efficiently implemented in the two-dimensional (2D) Fourier transformed domain. This article presents a novel sliding spotlight raw signal simulation approach from the widebeam SAR imaging modes. This approach can generate sliding spotlight raw signal not only from raw data evaluated by the simulators, but also from real data in the stripmap/spotlight mode. In order to obtain the desired raw data from conventional stripmap/spotlight mode, the azimuth time-varying filtering, which is implemented by de-rotation and low-pass filtering, is adopted. As raw signal of extended scenes in the stripmap/spotlight mode can efficiently be evaluated in the 2D Fourier domain, the proposed approach provides an efficient sliding spotlight SAR simulator of extended scenes. Simulation results validate this efficient simulator.
\end{abstract}

Keywords: synthetic aperture radar (SAR), sliding spotlight SAR, raw data simulator, extended scenes

\section{Introduction}

The spotlight synthetic aperture radar (SAR) mode can improve azimuth resolution by increasing the synthetic aperture time, and its azimuth beam is steered during the whole acquisition time. The major drawback of such configuration is that azimuth beam pointing always at the same area limits the extension of the illuminated area in azimuth. The sliding spotlight mode allows a comprise between azimuth resolution and azimuth extension of imaged scene [1-3], and it can be described as using a virtual rotation center which is further away from the radar than the imaging scene [4].

A SAR raw data simulator is an important tool for testing system parameters, imaging algorithms, and mission planning. SAR raw data can precisely be generated target by target in the two-dimensional (2D) time domain [5]. However, to the raw data simulation of extended scenes, this approach is of low efficiency. In order to improve the efficiency of raw data simulator, a series of simulators for different imaging modes are proposed in the 2D Fourier domain [5-9]. Unfortunately,

\footnotetext{
* Correspondence: iecasxuwei@gmail.com

${ }^{1}$ Institute of Electronics, Chinese Academy of Sciences (IECAS), Lab 6\#, No.

19, West Bei-Si-Huan Road, Hai Dian District, Beijing 100190, China

Full list of author information is available at the end of the article
}

different from the stripmap and the pure spotlight modes, the raw data of extended imaged scenes in the sliding spotlight mode may not efficiently be evaluated in the 2D Fourier transformed domain [10]. The 1D range Fourier domain approach for the sliding spotlight mode is proposed in [10], but it is still less efficient, compared with the 2D Fourier domain simulators.

This article presents a novel sliding spotlight SAR raw data generation approach from the wide-beam imaging modes. Azimuth varying band-pass filter (BPF) could be adopted for extracting the desired signals from the raw data in the wide-beam imaging modes and set others to zero. This filter should just accommodate the Doppler centroid varying rate and be independent of the slant range, and it can efficiently be implemented by de-rotation operation and BPF. This article is organized as follows: Section 2 reviews the acquisition geometry of the sliding spotlight mode and analyzes its special echo characteristics in azimuth. Section 3 is focused on presenting the proposed raw data generation approach of extended scenes for the sliding spotlight mode. Simulation results are given to validate the proposed raw data generation approach in Section 4. Finally, some useful conclusions are reported. 


\section{Sliding spotlight mode implementation}

The stripmap and spotlight modes are the two wellknown SAR operating schemes. In the stripmap mode, antenna azimuth beam points along a fixed direction with respect to the flight path, while azimuth beam is steered during the whole acquisition interval in the spotlight mode. In this section, both acquisitions geometries and echo characteristics in the sliding spotlight mode are compared with conventional stripmap and spotlight modes.

Different from conventional stripmap mode with a fixed azimuth beam pointing direction, azimuth beam is steered from fore to aft during the whole acquisition time in the sliding spotlight mode. If we just exploit part of azimuth beam in the stripmap mode to illuminate the imaged area, whose direction depends on the azimuth location of the sensor platform, the equivalent azimuth beam steering and the equivalent sliding spotlight mode with a reduced azimuth beam will be obtained as shown in Figure 1. In the figure, $v$ denotes the effective velocity of the sensor on the imaging plane, $T$ is the duration of the whole acquisition time, $L_{\mathrm{s}}$ and $L_{\mathrm{ss}}$ are the effective azimuth extension of the imaged area in the stripmap mode and sliding spotlight mode, respectively. Assuming that azimuth beam angular interval is $\theta_{0}$ in the sliding spotlight configuration and $\theta_{1}$ in the stripmap mode, their effective azimuth extensions of imaged area are approximatively expressed as follows:

$$
L_{\mathrm{ss}}=L_{\mathrm{s}}=v T-\theta_{1} R_{\mathrm{c}}
$$

where $R_{\mathrm{c}}$ is the slant range from the sensor to the imaged area center. If we choose part of azimuth beam interval to imitate azimuth beam steering in the sliding spotlight mode as shown in Figure 1, then the equivalent azimuth beam rotation rate is as follows:

$$
\omega_{r}=\frac{\theta_{1}-\theta_{0}}{T}
$$

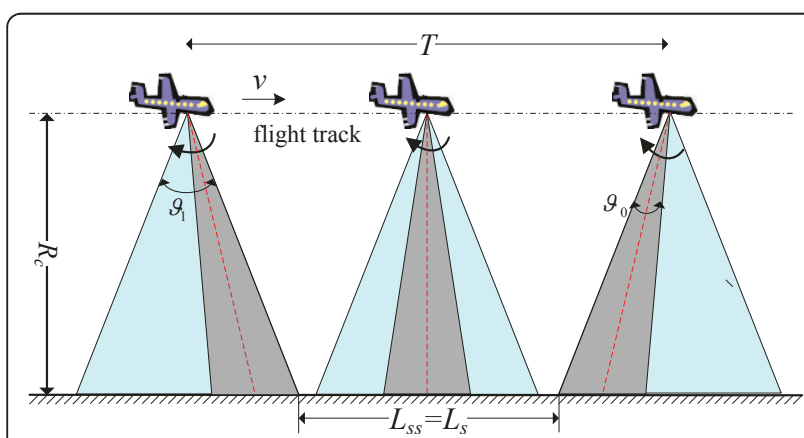

Figure 1 Sliding spotlight acquisition geometry implementation in the stripmap mode.
Similar to the pure spotlight mode, azimuth beam is steered from fore to aft during the overall acquisition time in the sliding spotlight mode. However, its azimuth beam is steered with a lower beam rotation rate. Therefore, the equivalent sliding spotlight configuration can also be implemented in the pure spotlight mode as shown in Figure 2. In the figure, $L_{\text {spot }}$ and $L_{\mathrm{ss}}$ are the effective azimuth extension of imaged area in the spotlight mode and sliding spotlight mode, respectively, and they are expressed as follows

$$
\begin{aligned}
& L_{\text {spot }}=\theta_{2} R_{\mathrm{c}} \\
& L_{\mathrm{ss}}=L_{\text {spot }}-2 \theta_{0} R_{\mathrm{c}}=\left(\theta_{2}-2 \theta_{0}\right) R_{\mathrm{c}}
\end{aligned}
$$

where $\theta_{2}$ is the azimuth beam angular interval in the spotlight mode. Thereafter, the equivalent azimuth beam rotation rate in Figure 2 can be obtained as follows

$$
\omega_{r}=\frac{v}{R_{\mathrm{c}}}-\frac{\theta_{2}-\theta_{0}}{T}
$$

The raw data support in the azimuth time/frequency domain (TFD) is shown in Figure 3. Different from stripmap/spotlight mode, both target Doppler centroid and beam center time vary with the target azimuth location. The TFD supports of the equivalent sliding spotlight are represented by the shaded blocks as shown in Figure 3. The dashed lines in Figure 3a,b indicate the target Doppler histories in the stripmap and spotlight modes, respectively. The solid thick lines denote the Doppler histories of the desired echoes in the equivalent sliding spotlight mode. The equivalent azimuth beam steering introduces a Doppler centroid rate as follows

$$
k_{\mathrm{rot}}=\frac{2 v \omega_{r}}{\lambda}
$$

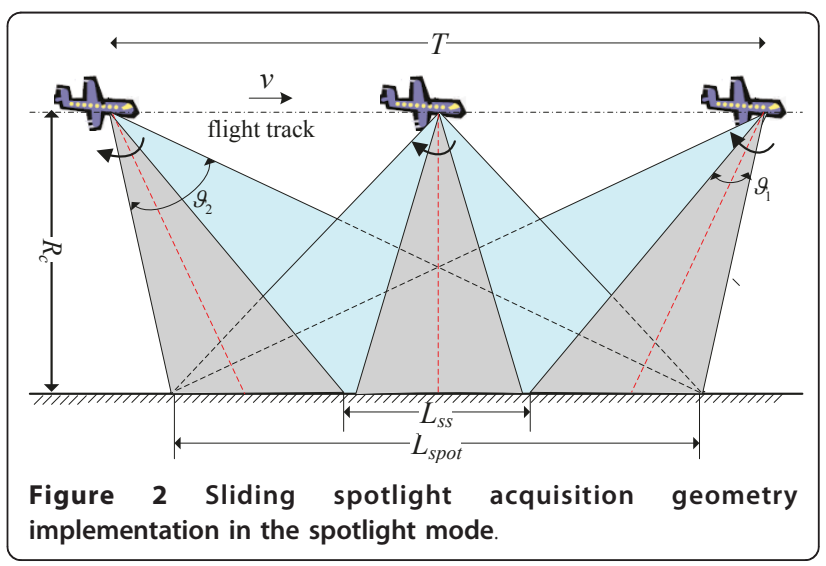




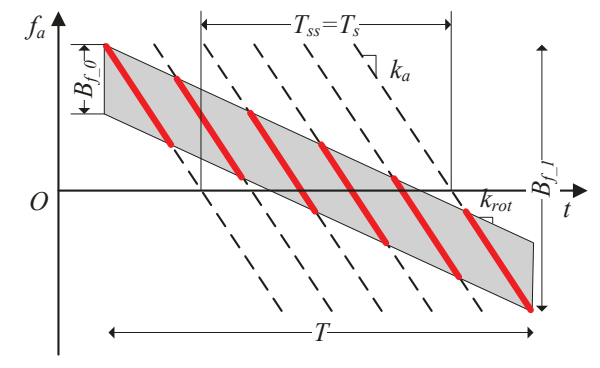

(a)

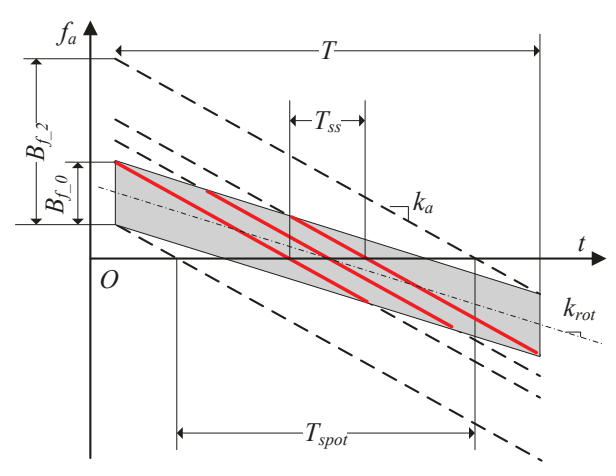

(b)

Figure 3 TFD supports in the equivalent sliding spotlight acquisition. Raw data evaluated from the (a) stripmap mode and (b) the spotlight mode.

where $\lambda$ is the wavelength. In Figure $3, k_{\mathrm{a}}$ is the target Doppler rate, $T_{\mathrm{s}}, T_{\mathrm{ss}}$, and $T_{\mathrm{spot}}$ are the effective azimuth output extensions of the stripmap mode, the equivalent sliding spotlight mode, and the pure spotlight mode, respectively. In the figure, $B_{f_{-}}, B_{f_{-} 1}$, and $B_{f_{-} 2}$ are the azimuth beam bandwidths in the equivalent sliding spotlight, stripmap, and the spotlight modes, respectively. The overall Doppler bandwidth of the equivalent sliding spotlight mode is as follows

$$
B_{s}=\left|k_{\mathrm{rot}}\right| T+B_{f_{-} 0}
$$

\section{Raw data generation approach}

From Figures 1, 2, and 3, we can see that the equivalent sliding spotlight SAR raw data can be extracted from the wide-beam stripmap/spotlight mode. If we remain the desired Doppler bandwidth in the equivalent sliding spotlight mode for each target and set others to zero, then the desired echoes will be obtained. The azimuth time-frequency variant filter can implement this operation $[11,12]$. The proposed sliding spotlight SAR raw data generation approach is summarized as follows:
(1) Assuming that azimuth extension of the designed extended scene is $L_{\mathrm{ss}}$, azimuth beam interval is $\theta_{2}$, and the obtained azimuth resolution is $\rho_{\mathrm{az}}$, the equivalent azimuth beam rotation rate is computed as follows:

$$
\omega_{r}=\frac{v}{R_{\mathrm{c}}}\left(1-\frac{2 \theta_{0} \rho_{\mathrm{az}}}{\lambda}\right)
$$

Then, the azimuth acquisition duration is as follows:

$$
T=\frac{L_{\mathrm{ss}}+\theta_{0} R_{\mathrm{c}}}{v-\omega_{r} R_{\mathrm{c}}}
$$

Finally, the wide-beam angular interval $\theta_{1}$ exploited in the stripmap mode and $\theta_{2}$ in the spotlight mode are considered as follows:

$$
\begin{aligned}
& \theta_{1}=\omega_{r} T+\theta_{0} \\
& \theta_{2}=\frac{v T}{R_{\mathrm{c}}}+\theta_{0}-\omega_{r} T
\end{aligned}
$$

(2) After computing all the parameters used in the stripmap/spotlight mode SAR raw data generation, we can introduce the efficient 2D frequency domain simulators of extended scenes in the stripmap/spotlight mode to generate raw data [5-9].

(3) Extracting desired azimuth signal in the equivalent sliding spotlight mode, and reduce the azimuth sampling frequency with the designed system pulse repetition frequency (PRF).

Based on the fact that changing the Doppler rate shifts the signals or spectrums [13-15], there are two efficient ways for implementing azimuth varying filtering: operating in the azimuth time domain and in the Doppler frequency domain, as shown in Figure 4. The main steps of the first approach are given as follows:

(1) De-rotating azimuth data to remove the time-varying Doppler centroid of the desired Doppler spectrums. The de-rotation function is given as follows:

$$
h(t)=\exp \left[-j \pi k_{\mathrm{rot}}\left(t-t_{\mathrm{mid}}\right)^{2}\right]
$$

where $t_{\text {mid }}$ is the acquisition center time.

(2) Fourier transforming (FT) the de-rotated signals in the azimuth direction. It should be noted that up-sampling in azimuth may be required in the spotlight mode to avoid the Doppler spectrum aliasing.

(3) Low-pass filtering the azimuth signals, the bandwidth of the low-pass filter is $B_{f_{-} 0}$ equivalent to the bandwidth of the reduced azimuth beam interval adopted in the equivalent sliding spotlight mode. Afterward, we should resample the azimuth signals with the designed PRF by decreasing azimuth total samples in the Doppler domain. 


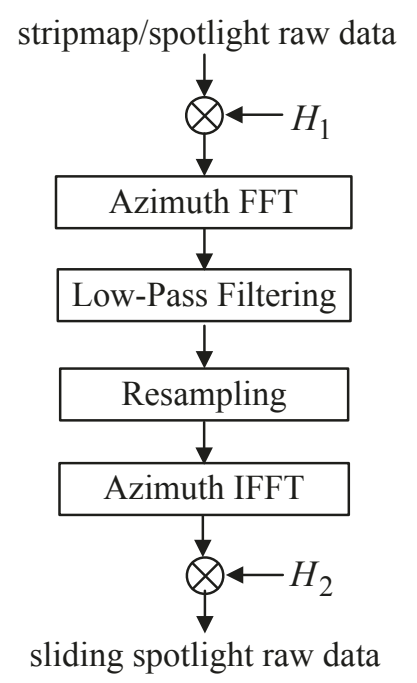

(a) Operating in the time domain

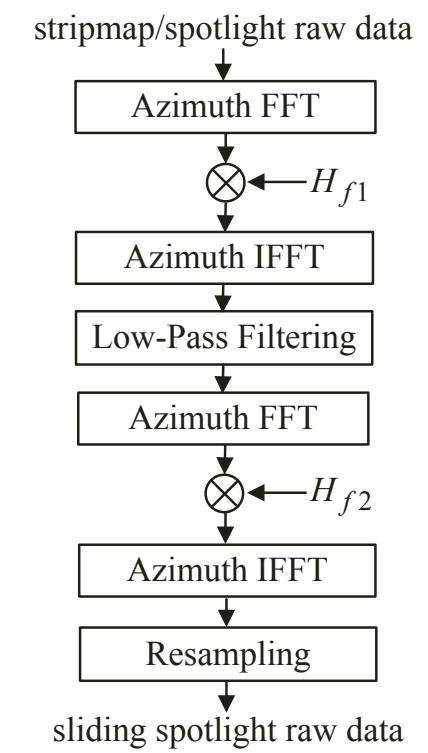

(b) Operating in the frequency domain

Figure 4 Flowcharts of proposed raw data generation approaches. Operating in the (a) time domain and (b) frequency domain.

(4) Inverse FT (IFT) the raw data in the azimuth direction and reramping of the azimuth data. The reramping referenced function is just conjugated to (12).

The second approach mainly implemented in the Doppler domain as shown in Figure 4b leads to the following processing steps:

(1) FT the raw data in the azimuth direction and low-pass filtering in the Doppler domain with the bandwidth $B_{\mathrm{w}}$ which is equivalent to the overall Doppler bandwidth $B_{s}$ in (7).

(2) Deramping the azimuth data in the Doppler domain to shift the azimuth signals, the referenced deramping function is given as follows:

$$
H(f)=\exp \left[j \pi\left(f_{a}-f_{\mathrm{sdc}}\right)^{2} / k_{\mathrm{rot}}\right]
$$

where $f_{a}$ is the Doppler frequency, and $f_{\text {sdc }}$ is the Doppler centroid of the illuminated scene.

(3) IFT the raw data in the azimuth direction and low-pass filtering the raw data in the azimuth time domain. The duration of the low-pass filter is $T_{\mathrm{w}}$ given by:

$$
T_{w}=\frac{B_{d}}{\left|k_{a} k_{\mathrm{rot}} /\left(k_{a}-k_{\mathrm{rot}}\right)\right|}
$$

where $k_{a}=-2 v^{2} /(\lambda r)$ is the Doppler rate and $B_{\mathrm{d}}$ is the target Doppler bandwidth in the sliding spotlight mode given by

$$
B_{\mathrm{d}}=\frac{B_{f_{-} 0}}{1-\omega_{r} r / v}
$$

where $r$ is the slant range. Substituting (15) into (14) yields:

$$
T_{w}=\frac{\lambda B_{f \_}}{2 v \omega_{r}}
$$

From (16), we can see that targets with different range bins require the same duration of the low-pass filter. Such phenomenon leads to the low-pass filtering be implemented more easily.

(4) FT the deramped signals in the azimuth direction and reramping of raw data by the referenced function which is conjugated to (13).

(5) IFT and resampling the raw data in the azimuth direction to obtain the desired sliding spotlight raw data.

Compared with the time domain approach, the Doppler domain approach is less efficient, as it requires two FT operations and two IFT operations while the time domain approach only needs one FT 


\section{Table 1 System parameters in the simulation}

\begin{tabular}{ll}
\hline Carrier frequency & $9.65 \mathrm{GHZ}$ \\
Platform velocity & $200 \mathrm{~m} / \mathrm{s}$ \\
Pulse duration & $1 \mu \mathrm{s}$ \\
Pulse bandwidth & $100 \mathrm{MHz}$ \\
Sampling frequency & $120 \mathrm{MHz}$ \\
Antenna length in the wide-beam mode & $0.5 \mathrm{~m}$ \\
Antenna length in the sliding spotlight mode & $2 \mathrm{~m}$ \\
PRF in the wide-beam mode & $1000 \mathrm{~Hz}$ \\
PRF in the sliding spotlight mode & $250 \mathrm{~Hz}$ \\
Slant range & $10 \mathrm{~km}$ \\
The desired azimuth resolution in the sliding spotlight mode & $0.8 \mathrm{~m}$
\end{tabular}

operation and one IFT operation in azimuth, as shown in Figure 4.

\section{Simulation experiment}

To validate the proposed approach, a raw data simulation experiment is taken. In this simulation, the parameters are listed in Table 1.

The designed imaged scene consisting of five-point targets is shown in Figure 5a, and its raw data in the stripmap mode are shown in Figure 5b. With the proposed raw data generation approach, the sliding spotlight SAR raw data obtained from Figure $5 \mathrm{~b}$ is shown in Figure 5c, and its imaging result is shown in Figure $5 \mathrm{~d}$. The difference between the phase of the raw signal simulated by using the proposed approach and the phase of the raw signal simulated by using the traditional approach in the $2 \mathrm{D}$ time domain in azimuth is shown in Figure 6a, and the amplitudes of the raw signal in azimuth are considered in Figure 6b. Clearly, we can see that the absolute value of the phase difference is always much smaller than 0.4 rad, which leads to negligible effects. Moreover, small amplitude oscillations around the exact constant values caused by FT operation in the discrete domain can be noted.

In the simulation of extended scenes, we use a focused SAR image, the size of which is $150 \times 150$ pixels, as a scattering coefficient matrix, as shown in Figure 7a. The distance between two adjoining pixels in both azimuth and range direction is $0.5 \mathrm{~m}$. Taking the scattering coefficient matrix, the efficient 2D Fourier domain simulator in [6] is adopted for generating the stripmap SAR raw data and its imaging result is shown in Figure $7 \mathrm{~b}$. With the proposed sliding spotlight SAR raw data generation approach, we obtain the equivalent sliding spotlight SAR

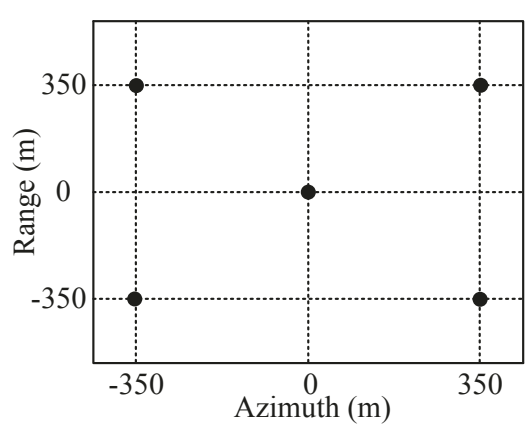

(a) The designed imaged scene.

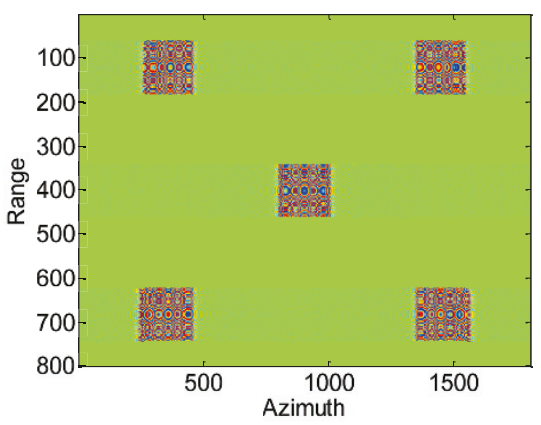

(c) The evaluated sliding spotlight raw data.

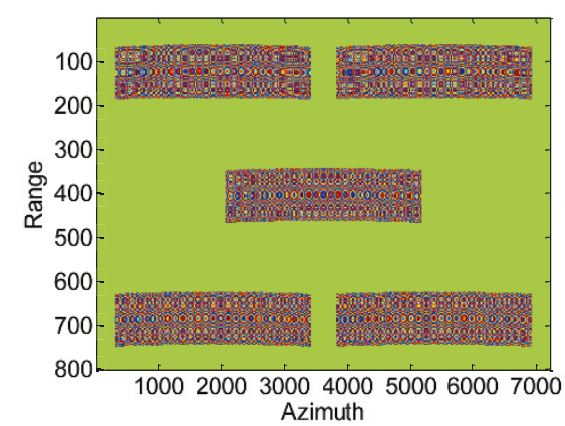

(b) Raw data in the stripmap mode.

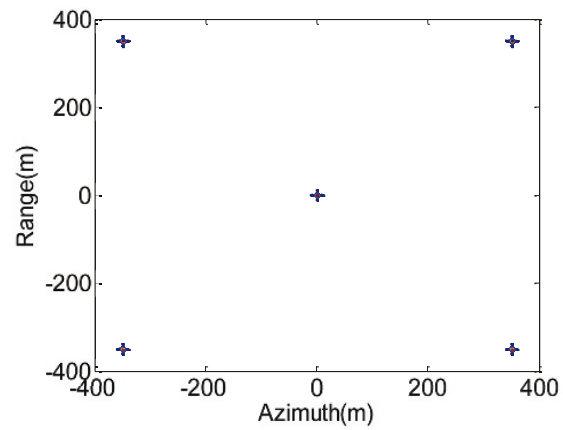

(d) Imaging result of the sliding spotlight raw data.

Figure 5 The sliding spotlight SAR signals simulation of point targets. (a) The designed imaged scene. (b) Raw data in the stripmap mode. (c) The evaluated sliding spotlight raw data. (d) Imaging result of the sliding spotlight raw data. 


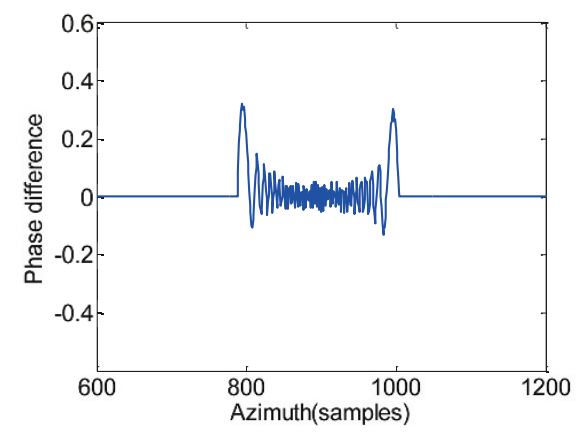

(a) Phase difference.

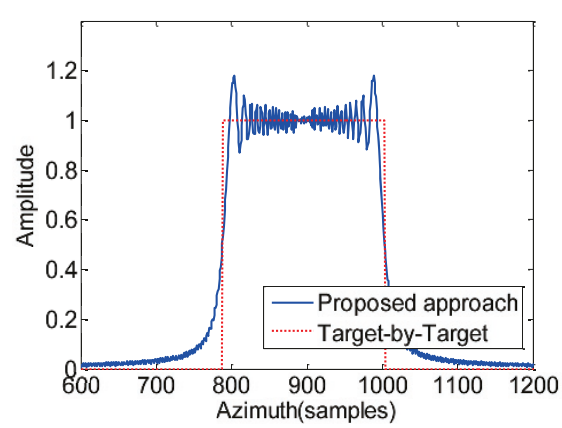

(b) Amplitudes of the raw signals.

Figure 6 Phase difference and amplitude of the simulated raw signals in azimuth. (a) Phase difference and (b) amplitudes of the raw signals.

raw data, in which we implement the azimuth timevarying filtering in the time domain. Figure $7 \mathrm{c}$ shows the equivalent sliding spotlight SAR raw data, and its imaging result is shown in Figure $7 \mathrm{~d}$. Clearly, the simulation results of Figures 5 and 7 validate the proposed raw data generation approach.

\section{Conclusion}

This article presents a novel approach to generate the sliding spotlight SAR raw data from the wide-beam stripmap/spotlight mode. As both stripmap and spotlight SAR raw data of extended scenes can efficiently be generated in the 2D Fourier transformed domain [5-9],

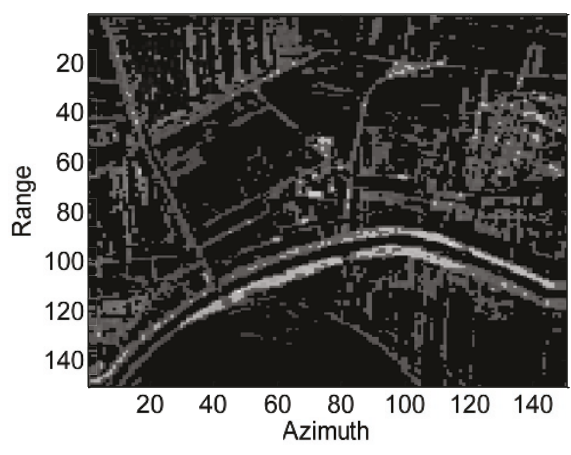

(a) Scattering coefficient matrix of the imaged scene.

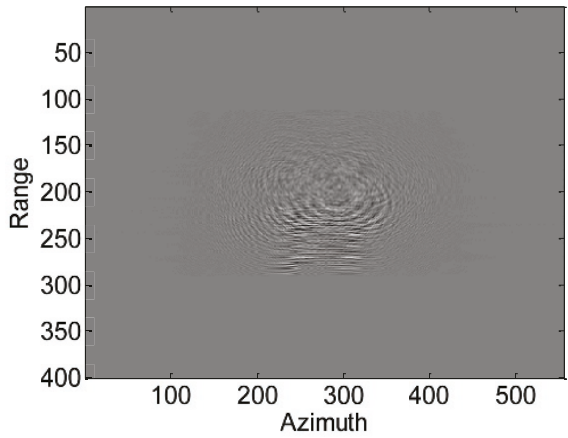

(c) The simulated sliding spotlight raw data.

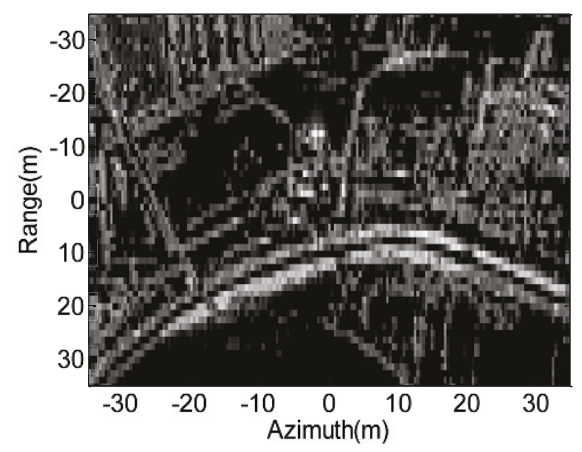

(b) Imaging result of simulated raw data in the stripmap mode.

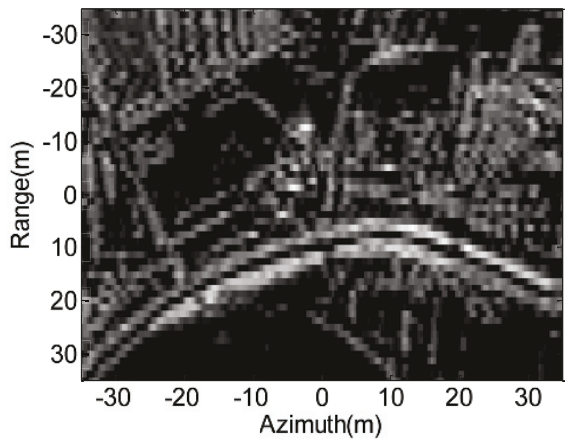

(d) Imaging result of the sliding spotlight raw data.

Figure 7 Simulation of the extended scene. (a) Scattering coefficient matrix of the imaged scene. (b) Imaging result of simulated raw data in the stripmap mode. (c) The simulated sliding spotlight raw data. (d) Imaging result of the sliding spotlight raw data. 
this approach provides an efficient real-time sliding spotlight raw data simulator of extended scenes. From Figure 4, it can be seen that this approach is quite efficient, owning to the fact that only complex multiplications and FFT codes are needed. Furthermore, the equivalent sliding spotlight raw data can also be obtained from real data of the wide-beam imaging modes.

\section{Author details}

'Institute of Electronics, Chinese Academy of Sciences (IECAS), Lab 6\#, No. 19, West Bei-Si-Huan Road, Hai Dian District, Beijing 100190, China ${ }^{2}$ College of Information Engineering, Inner Mongolia University of Technology, Hohhot 010051, China

\section{Competing interests}

The authors declare that they have no competing interests.

Received: 8 December 2010 Accepted: 5 September 2011 Published: 5 September 2011

\section{References}

1. DP Belcher, CJ Baker, High resolution processing of hybrid stripmap/ spotlight mode SAR, in Proc Inst Elect Eng, Radar Sonar Navigat. 143, 366-374 (1996). doi:10.1049/ip-rsn:19960790

2. G Fornaro, R Lanari, E Sansosti, F Serafino, S Zoffoli, A new algorithm for processing hybrid strip-map/spotlight mode synthetic aperture radar data, in Proc SPIE. 4173, 17-28 (2000)

3. R Lanari, S Zoffoli, E Sansosti, F Serafino, New approach for hybrid stripmap/spotlight SAR data focusing. IEE Proc Radar Sonar Navigat. 148(6), 363-372 (2001). doi:10.1049/ip-rsn:20010662

4. J Mittermayer, RT Lord, E Boerner, Sliding spotlight SAR processing for TerraSAR-X using a new formulation of the extended chirp scaling algorithm, in Proc IGARSS (Toulose, France, 2003), pp. 1462-1464

5. X Qiu, D Hu, L Zhou, C Ding, A bistatic SAR raw data simulator based on inverse $\omega$-k algorithm. IEEE Trans Geosci Remote Sens. 48(3), 1540-1547 (2010)

6. G Franceschetti, M Migliaccio, D Riccio, G Schirinzi, SARAS: a SAR raw signal simulator. IEEE Trans Geosci Remote Sens. 30(1), 110-123 (1992). doi:10.1109/36.124221

7. S Cimmino, G Franceschetti, A lodice, D Riccio, G Ruello, Efficient spotlight SAR raw signal simulation of extended scenes. IEEE Trans Geosci Remote Sens. 41(10), 2329-2337 (2003). doi:10.1109/TGRS.2003.815239

8. Y Wang, Z Zhang, Y Deng, Squint spotlight SAR raw signal simulation in the frequency domain using optical principles. IEEE Trans Geosci Remote Sens. 46(8), 2208-2215 (2008)

9. G Franceschetti, A lodice, S Perna, D Riccio, Efficient simulation of airborne SAR raw data of extended scenes. IEEE Trans Geosci Remote Sens. 44(10), 2851-2860 (2006)

10. G Franceschetti, R Guida, A lodice, D Riccio, G Ruello, Efficient simulation of hybrid stripmap/spotlight SAR raw signals from extended scenes. IEEE Trans Geosci Remote Sens. 42(10), 2385-2396 (2004)

11. A Monti Guarnieri, C Prati, ScanSAR focusing and interferometry. IEEE Trans Geosci Remote Sens. 34(4), 1029-1038 (1996). doi:10.1109/36.508420

12. C Prati, A Monti Guarnieri, F Rocca, Spot mode SAR focusing with the omega-k technique, in Proc IGARSS (Helsinki, Finland, 1991), pp. 631-634

13. P Prats, R Scheiber, J Mittermayer, A Meta, A Moreira, Processing of sliding spotlight and TOPS SAR data using baseband azimuth scaling. IEEE Trans Geosci Remote Sens. 48(2), 770-780 (2010)

14. $W X u, P$ Huang, Y Deng, TOPSAR data focusing based on azimuth scaling preprocessing. Adv Space Res. 48(2), 270-277 (2011). doi:10.1016/j. asr.2011.03.024

15. W Xu, P Huang, Y Deng, An efficient imaging approach with scaling factors for TOPS mode SAR data focusing. IEEE Geosci Remote Sens Lett. 8(5), 929-933 (2011) doi:10.1186/1687-6180-2011-52

Cite this article as: Xu et al:: Efficient sliding spotlight SAR raw signal simulation of extended scenes. EURASIP Journal on Advances in Signal Processing 2011 2011:52.

\section{Submit your manuscript to a SpringerOpen ${ }^{\mathcal{O}}$ journal and benefit from:}

- Convenient online submission

- Rigorous peer review

- Immediate publication on acceptance

- Open access: articles freely available online

- High visibility within the field

- Retaining the copyright to your article

Submit your next manuscript at $\gg$ springeropen.com 